\title{
IMPROVED TIME DIVERSITY FOR LTE OVER SATELLITE USING SPLIT MULTICODE TRANSMISSION
}

Adegbenga B. Awoseyila and Barry G. Evans

Centre for Communication Systems Research (CCSR),

University of Surrey, Guildford GU2 7XH, U.K.

a.awoseyila@surrey.ac.uk,b.evans@surrey.ac.uk

An improved time diversity technique is proposed for deploying the 3GPP LTE airinterface over satellite mobile links. It overcomes the FEC performance loss resulting from the use of a short transmit-time-interval (TTI) in LTE. By using transport block expansion, code block segmentation, advanced rate matching and automatic retransmissions, the FEC coded block is split over as many TTIs as is necessary to break the channel correlation without any reduction in the user data rate.

Introduction: Terrestrial mobile operators find it difficult to cover rural and remote areas since the high cost of infrastructure deployment is not adequately justified by the low population density. In this regard, satellite mobile systems have an advantage due to their inherently wide coverage area and can be used to complement terrestrial mobile services. An integrated approach with maximum commonality in air-interface will help to minimize service delivery costs to the user. A strong candidate air-interface for $4 G$ mobile communications is the 3GPP LTE standard [1]. It uses a short transmit time interval (TTI) of $1 \mathrm{~ms}$ which helps to support low-latency and fast channel-aware scheduling, thereby increasing throughput [2].

In contrast to terrestrial systems which can effectively combat fast-fading using link adaptation techniques such as 'Adaptive Modulation and Coding' and Hybrid Automatic Repeat request (HARQ), the large propagation delay in satellite systems inhibits fast link adaptation. For example, the round trip delay (RTD) of terrestrial links is practically less than $1 \mathrm{~ms}$ while it is $\sim 500 \mathrm{~ms}$ for a GEO satellite. On the other hand, a short TTI implies that the satellite mobile link cannot achieve significant 
channel interleaving gain. A method proposed in [3] to tackle this problem through forced retransmission and inter-TTI interleaving results in a reduction in user data rate and also contends with non-conformity to the LTE resource block structure.

In order to address these drawbacks, we propose a hybrid technique that reuses existing mechanisms in LTE to achieve sufficient time diversity to combat fast-fading in satellite links whilst complying fully with LTE resource block/TTI structure. The original user data rate is maintained at the expense of a linear increase in latency and memory requirements.

LTE Physical Layer: The LTE standard is designed for high data rate mobile communications. It implements a basic rate 1/3 parallel concatenated convolutional (turbo) code for forward error control (FEC). A rate matching stage processes the coded bits (using sub-block interleaving and interlacing) into a virtual circular buffer from which they are read out according to the specified 'rate-matched' coded block size and redundancy version [4]. Where a transport block is longer than the maximum input code block size, it is segmented into multiple code blocks which undergo independent FEC coding and rate-matching. The final codeword is then a concatenation of the different rate-matched coded blocks [2],[4].

After the FEC stage, the final codeword is scrambled and mapped to a QAM signal constellation, multiplexed to subcarriers using the LTE resource block/TTI structure and transmitted using OFDM. The minimum unit of resource allocation in LTE is a resource block which consists of 12 consecutive frequency subcarriers over 6 or 7 consecutive OFDM symbols. Resource blocks are usually allocated in pairs to form a subframe whose duration is equivalent to the LTE TTI of $1 \mathrm{~ms}$.

LTE supports incremental redundancy HARQ which consists of transmitting different parts of the FEC coded block in every retransmission such as to increase the FEC protection for packets in error. In a good channel, the original code block can be transmitted with no error protection, leading to increased throughput [5]. 
Proposed Technique: It is proposed that the initial transport block (corresponding to an initial code block size: $K$ ) be expanded in geometric proportion to the desired order of time diversity: $S$ for an FEC code block as shown in (1). The expanded code block is then split into $S$ multiple code blocks, each having a size equal to the initial code block size: $K$. Each code block then undergoes rate 1/3 FEC coding independently, resulting in a complete coded block size: $N$ as shown in (2). Advanced rate-matching is then performed per coded block in proportion to the desired overall code rate: $\rho$ and order of time diversity: $S$ as shown in (3). In this regard, a rate-matched coded block can be (significantly) shorter than the original code block. The final codeword is then a concatenation of the different rate-matched coded blocks corresponding to the different code block segments as shown in (4).

$$
\begin{aligned}
& \alpha=S K \\
& N \approx 3 K \\
& \beta=K /(S \rho) \\
& \delta=S \beta=K / \rho
\end{aligned}
$$

where $\alpha$ is the expanded code block size (resulting from the expanded transport block), $\beta$ is the rate-matched coded block size and $\delta$ is the final codeword size.

Automatic retransmissions based on incremental redundancy are then used to complete the transmission of each FEC coded block ( $S$ transmissions needed). The number of parallel HARQ processes: $P$ is set such as to break the channel correlation between retransmissions as shown in (5)-(7).

$$
\begin{aligned}
& f_{d m}=f_{c} v / c \\
& T_{c} \approx 1 /\left(2 f_{d m}\right)
\end{aligned}
$$


$P=\left(T_{c} / T_{T T I}\right)-1$

where $T_{c}$ is the channel coherence time and $f_{d m}$ is the maximum Doppler frequency shift [2] resulting from mobile speed $v$, carrier frequency $f_{c}$ and speed of light $c$. $T_{T T I}$ is the transmit time interval.

Table 1 illustrates the proposed method, wherein each FEC coded block is split into $S$ partitions via advanced rate matching, with each partition being transmitted $P$ TTIs apart, thereby achieving time diversity of the order $S$. Parallel HARQ processes and multicode transmission ensure that the user data rate is maintained at the expense of latency and memory requirements which increase in direct proportion to $S P$. However, memory is increasingly affordable and the additional latency is mostly a fraction of the satellite RTD.

Performance Evaluation: Computer simulations have been used to verify the performance of the proposed method for LTE downlink based on the following parameters: carrier frequency: $2 \mathrm{GHz}$, bandwidth: $5 \mathrm{MHz}$, mobile speed: $30 \mathrm{~km} / \mathrm{h}$, subcarrier spacing: $15 \mathrm{KHz}, \mathrm{FFT}$ size: 512, CP length: 128 and TTI duration: $1 \mathrm{~ms}$. QPSK modulation implies $\delta=6800$ data bits and a code block size $K=2304$ corresponds to a desired overall code rate of $\rho \approx 0.34$ and a user data rate of $R \approx 2.3 M b p s$. The channel is modelled based on the MAESTRO profile [6] for satellite transmission in outdoor rural areas as shown in Table 2. $P=8$ parallel HARQ processes are implemented based on a channel coherence time of $T_{c} \approx 9 \mathrm{~ms}$. Figure 1 shows the significant improvement in block error rate (BLER) achieved by the proposed 'Split LTE' as compared to the conventional 'Default LTE'. For a target BLER $=10^{-3}$, using $S=3$ (re)transmissions results in $\sim 4 \mathrm{~dB}$ gain in required $E_{b} / N_{0}$ which increases to $\sim 6 \mathrm{~dB}$ for $S=12$ (re)transmissions. $S=24$ achieves only $\sim 0.5 \mathrm{~dB}$ 
additional gain, indicating optimisation of time diversity in the channel. These improvements in link performance are achieved without a geometric reduction in user data rate of the order $S$. As seen in the results, quasi-error-free performance is achieved for 12 (re)transmissions at $E_{b} / N_{0}<4 d B$, resulting in a link budget gain greater than $6 \mathrm{~dB}$. This implies a capacity increase of more than 4 times the default LTE capacity for a satellite mobile service.

\section{Conclusions:}

An improved time diversity technique has been proposed for short TTI transmissions. The method has been shown to achieve significant time diversity gain in a wideband fading channel without reduction in the user data rate, while maintaining maximum commonality with the LTE standard. The gains achieved will allow reasonable capacities that had hitherto not been possible with satellite mobile systems.

\section{Acknowledgements:}

This work was funded in part by the European SatNEx programme and ETRI, Korea.

References:

1. 3GPP TS 36.211 v8.5.0, 3rd Generation Partnership Project; Technical Specification Group Radio Access Network; Evolved Universal Terrestrial Radio Access (E-UTRA); Physical Channels and Modulation (Release 8), 2008.

2. Sesia S., Toufik I., and Baker M., LTE, The UMTS Long Term Evolution: From Theory to Practice, John Wiley and Sons, 2009.

3. Papaleo M., Neri M., Vanelli-Coralli A., and Corazza G. E., "Using LTE in 4G satellite communications: Increasing time diversity through forced retransmission," 10th Intl. Workshop on Signal Process. Space Commun. (SPSC), pp. 1-4, Oct. 2008. 
4. 3GPP TS 36.212 v8.5.0, $3^{\text {rd }}$ Generation Partnership Project; Technical Specification Group Radio Access Network; Evolved Universal Terrestrial Radio Access (E-UTRA); Multiplexing and channel coding (Release 8), 2008.

5. Beh K.C., Doufexi, A., and Armour, S., "Performance evaluation of hybrid ARQ schemes of 3GPP LTE OFDMA system", 18th Intl. Personal, Indoor and Mobile Radio Communications, (PIMRC), pp. 1-5, Sept. 2007.

6. "Mobile Applications and sErvices based on Satellite and Terrestrial inteRwOrking (IST-2002-507023)", EC FP6 MAESTRO project. 


\section{Figure/Table Captions}

Table 1: $\quad$ Proposed architecture for split multicode transmission

Table 2: $\quad$ Power-Delay-Profile for satellite wideband channel

Figure 1: $\quad$ BLER performance for Split LTE in satellite wideband channel 
Table 1

\begin{tabular}{|c|c|c|c|c|}
\hline \multirow{7}{*}{ 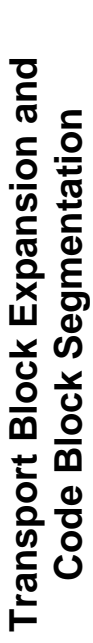 } & $\begin{array}{l}\text { Coded Block } \\
\text { per Segment }\end{array}$ & \multirow{7}{*}{ 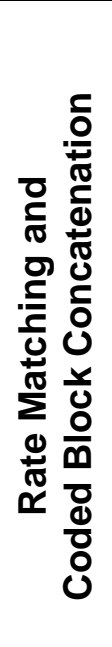 } & $\begin{array}{c}\text { Transmit Block } \\
\text { per TTI }\end{array}$ & TTI \# \\
\hline & $A_{1,1}, A_{1,2}, \cdots, A_{1, S}$ & & $A_{1,1}, A_{2,1}, \cdots, A_{S, 1}$ & 0 \\
\hline & $A_{2,1}, A_{2,2}, \cdots, A_{2, S}$ & & $A_{1,2}, A_{2,2}, \cdots, A_{S, 2}$ & $1 P$ \\
\hline & • & & . & $\cdot$ \\
\hline & • & & . & $\cdot$ \\
\hline & $\cdot$ & & $\cdot$ & $\cdot$ \\
\hline & $A_{S, 1}, A_{S, 2}, \cdots, A_{S, S}$ & & $A_{1, S}, A_{2, S}, \cdots, A_{S, S}$ & $(S-1) P$ \\
\hline
\end{tabular}


Table 2

\begin{tabular}{|c|c|c|c|c|c|c|c|}
\hline Tap number & 1 & 2 & 3 & 4 & 5 & 6 & 7 \\
\hline $\begin{array}{c}\text { Relative mean power } \\
(\mathrm{dB})\end{array}$ & 0 & -14.4 & -18.2 & -20.6 & -18.3 & -20.6 & -20.6 \\
\hline Relative delay (ns) & 0 & 195.3 & 260.4 & 846.3 & 1171.9 & 1953.1 & 2734.3 \\
\hline K-factor (dB) & 10 & -inf & -inf & - inf & -inf & -inf & -inf \\
\hline
\end{tabular}




\section{Figure 1}

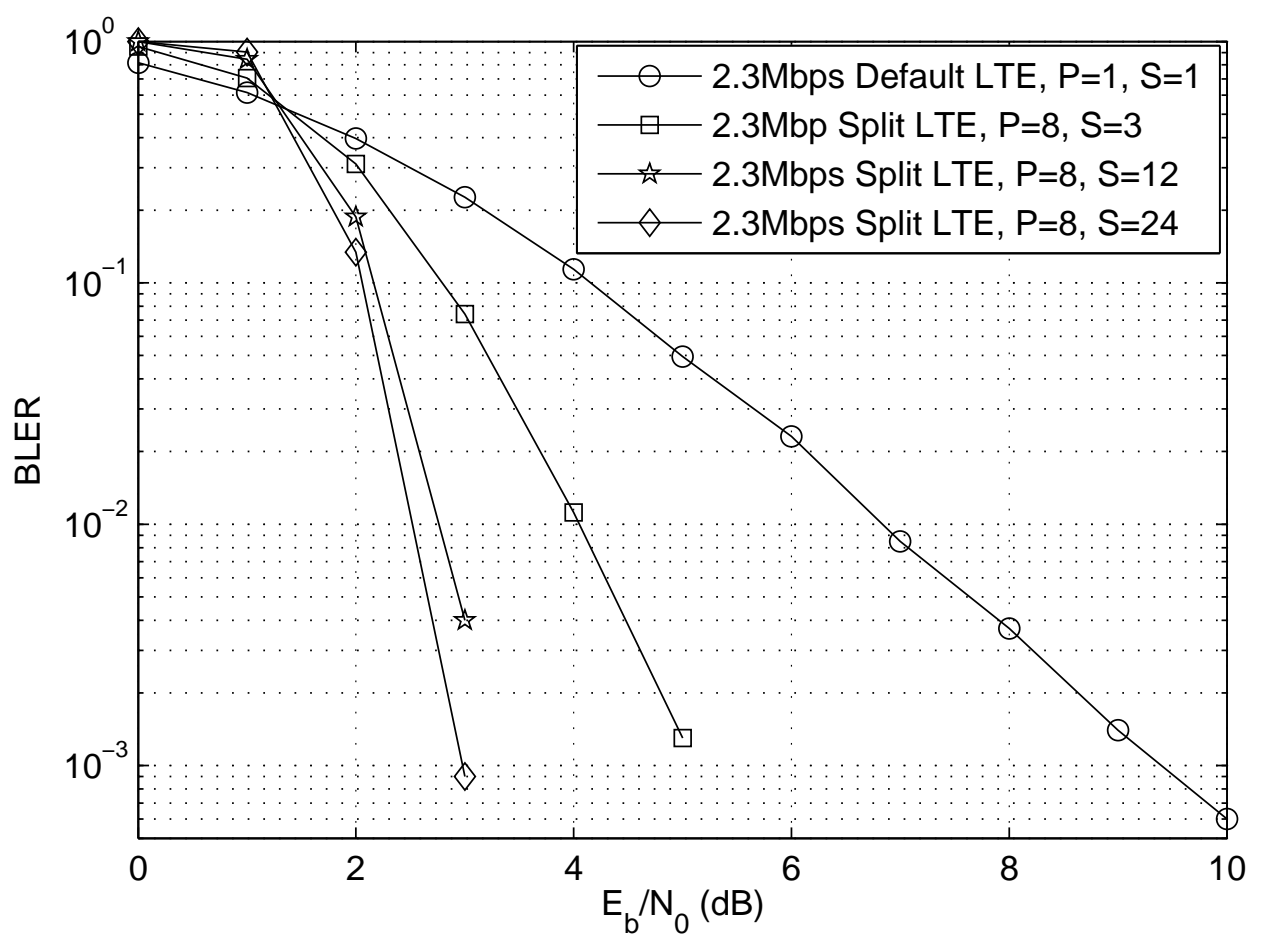

